\title{
Neural Correlates of Visual versus Abstract Letter Processing in Roman and Arabic Scripts
}

\author{
Manuel Carreiras $^{1,2}$, Manuel Perea ${ }^{3}$, Cristina Gil-López ${ }^{1}$, \\ Reem Abu Mallouh ${ }^{1}$, and Elena Salillas ${ }^{1}$
}

\begin{abstract}
In alphabetic orthographies, letter identification is a critical process during the recognition of visually presented words. In the present experiment, we examined whether and when visual form influences letter processing in two very distinct alphabets (Roman and Arabic). Disentangling visual versus abstract letter representations was possible because letters in the Roman alphabet may look visually similar/dissimilar in lowercase and uppercase forms (e.g., c-C vs. r-R) and letters in the Arabic alphabet may look visually similar/dissimilar, depending on their position within a word (e.g., $\dot{e}$ - ف vs. $\boldsymbol{c}$ - ع). We employed a masked priming same-different matching task while
\end{abstract}

\section{INTRODUCTION}

Letter identification is a fundamental part of the process of lexical access in alphabetic orthographies. Learning to read involves a thorough training in which the cognitive system associates a common (abstract) representation to the different visual forms in which a letter is presented (i.e., $\mathrm{d}, \mathbf{D}, \boldsymbol{d}$, or D would correspond to the abstract letter unit "d"). Indeed, a basic assumption of neural accounts of letter and word recognition (e.g., Grainger, Rey, \& Dufau, 2008; Dehaene, Cohen, Sigman, \& Vinckier, 2005; Whitney, 2001) is that letters are initially processed by detectors at a retinotopic level: Visually different letters across case like "g" and "G" would activate different letter detectors, whereas visually similar letters across case like "c" and "C" would activate the same detectors. Later in processing, the letters would be processed at an abstract level (i.e., invariant to visual factors) so that visually different letters across case like "g" and "G" would also activate the same letter detectors.

To track the time course of visual versus abstract representations of letters, an excellent approach is to employ a technique that taps into the early stages of processing such as masked priming (Forster \& Davis, 1984; see Grainger et al., 2008, for a review) while ERPs are being

\footnotetext{
${ }^{1}$ Basque Center for Cognition, Brain, and Language, DonostiaSan Sebastián, Spain, ${ }^{2}$ IKERBASQUE, Basque Foundation for Science, Bilbao, Spain, ${ }^{3}$ Universitat de València, Valencia, Spain
}

ERPs were measured from individuals who had learned the two alphabets at an early age. Results revealed a prime-target relatedness effect dependent on visual form in early components $(\mathrm{P} / \mathrm{N} 150)$ and a more abstract relatedness effect in a later component (P300). Importantly, the pattern of data was remarkably similar in the two alphabets. Thus, these data offer empirical support for a universal (i.e., across alphabets) hierarchical account of letter processing in which the time course of letter processing in different scripts follows a similar trajectory from visual features to visual form independent of abstract representations. recorded. Empirical evidence using masked priming with behavioral measures does suggest that there is fast access to abstract letter representations. For the sake of simplicity, we will focus on the masked priming same-different matching task, which is the task employed in the present experiment (see Carreiras, Perea, \& Abu Mallouh, 2012, for a review of the literature on masked letter priming with other tasks). The typical setup of this task is the following: A probe is initially presented for $1 \mathrm{sec}$, after which the target item is preceded by a forwardly masked $50-\mathrm{msec}$ prime. The participant's task is to decide whether the target is the same as the probe (see Figure 1 for a depiction of the task). For "same" probe-target trials, Kinoshita and Kaplan (2008) found masked repetition priming effects of comparable magnitude for prime-target pairs that had similar and dissimilar visual features across case (e.g., c-C and g-G). Using a task/design parallel to that of Kinoshita and Kaplan (2008), Carreiras et al. (2012) found a similar masked repetition effect in an intricate script, Arabic. Arabic does not have the lowercase/uppercase distinction. Instead, the shape of the letters in Arabic may vary depending on the position in the word (initial, middle, final, isolated; see Carreiras et al., 2012, for further details). Specifically, some of the letters look visually dissimilar in the different word positions (e.g., $\rightarrow$ and $\boldsymbol{\varepsilon}$ correspond to the letter 'ayn), whereas others look visually similar (e.g., $\dot{i}$ and $\dot{i}$ correspond to the letter $\underline{\mathrm{fa}} \mathbf{})$. Carreiras et al. found that the magnitude of the masked repetition priming effect was equivalent for letter pairs with similar visual features 
Figure 1. Experimental paradigm.

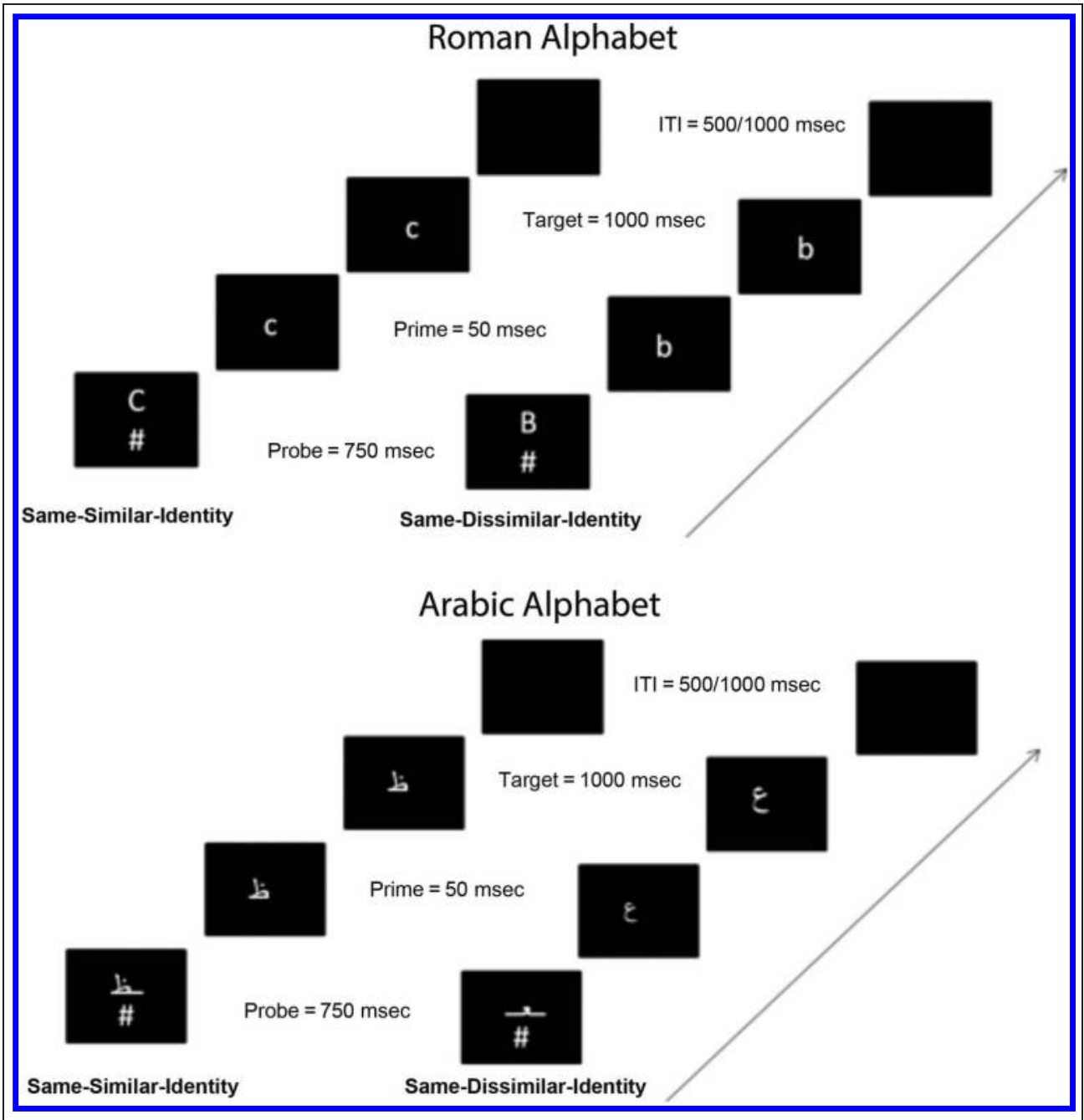

across word position and for letter pairs with dissimilar visual features across word position. Taken together, these experiments have shown that (i) the cognitive system quickly converts a letter's visual codes into abstract codes and (ii) this process occurs in very different orthographies (see also Perea, Abu Mallouh, \& Carreiras, 2013; Bowers, Vigliocco, \& Haan, 1998, for behavioral evidence of fast activation of abstract codes using masked priming with words in English and Arabic, respectively).

One obvious limitation of behavioral experiments on letter recognition is that early temporal differences in the visual processing of letters may not be reflected in RTs. Bear in mind that pairs of visually similar letters like "C" and "C" may produce greater relatedness effects early in processing (at perceptual, retinotopic level) than pairs of visually dissimilar letters like "g" and "G", but this may be hidden by a similar activation later in processing (at a more abstract level). To investigate in detail the time course of letter perception and thus to disentangle (potential) early effects of visual letter form and later effects of abstract letter identities, the best strategy is to obtain an on-line measure of letter processing. One such option is to record ERPs - these are voltage changes recorded from the scalp and extracted from the background EEG by averaging time-locked responses to stimuli onset.

To our knowledge, there is only one published experiment on the time course of letter processing that combined masked priming with ERP recordings. Petit, Midgley, Holcomb, and Grainger (2006) presented singleletter targets that had been preceded by a briefly masked letter prime-they used the 26 letters in the Roman alphabet. Petit et al. manipulated the name and case consistency across primes and targets and also examined whether letters had uppercase and lowercase forms with similar or dissimilar features. No explicit task was required from the participants in each trial; but every six trials on average, the trial sequence was followed by a "recall" signal ("?") and participants had to press the key corresponding to the target letter they had just seen. Petit et al. found an effect of prime-target visual similarity between 120 and 180 msec. Given that visual similarity was confounded with differences in name and case, they contrasted primetarget letter pairs of the same name conducting an analysis as a function of the similarity of the uppercase and 
lowercase versions of the letters and found that the P150 component was larger for dissimilar letters. Petit et al. also reported an interaction between case and name consistency, which they interpreted as an effect of case-specific letter identity between 180 and $220 \mathrm{msec}$, although no analyses were reported concerning the visual similarity between lowercase and uppercase versions of the same letter in this time window. Finally, they found an effect of case-independent letter identity between 220 and $300 \mathrm{msec}$. Petit et al. discussed their data as supporting a hierarchical account of letter recognition that involves both early case-specific and late case-independent representations of letters.

Other ERP experiments have also examined the impact of visual similarity on letter recognition using nonpriming paradigms. Rey, Dufau, Massol, and Grainger (2009) found differences between letters and matched nonletters in visual features around $150 \mathrm{msec}$ after stimulus onset (see also Wong, Gauthier, Woroch, DeBuse, \& Curran, 2005; Bentin, Allison, Puce, Perez, \& McCarthy, 1996, for similar findings in a similar time window [N170]). Finally, Madec, Rey, Dufau, Klein, and Grainger (2012) examined the correlation between interitem variability in behavioral and ERP measures during letter processing. In an early time window (between 90 and $110 \mathrm{msec}$ ), they found significant correlations at occipital electrodes. Later, in a 150-200 msec time window, effects were significant at the occipital and fronto-central electrodes. Finally, they found significant correlations at occipital electrodes in the 200-250 msec time window. Madec et al. suggested that, consistent with a hierarchical model of letter recognition, low-level visual feature processing is observed at occipital regions around $100 \mathrm{msec}$, followed by letter identification processes between 150 and $200 \mathrm{msec}$ in more anterior regions and feedback processing participating in the conscious identification of the letter in primary visual areas in the 200-250 msec time window.

The hierarchical (neurologically plausible) accounts of letter/word recognition proposed by Grainger et al. (2008) and Dehaene et al. (2005) were originally designed for the Roman alphabet. One basic question here is whether these accounts are universal or not and so hold across alphabets. This is particularly important because the different visual forms in the two alphabets studied here have different origins: case in the Roman alphabet versus position within a word in the Arabic alphabet. Understanding the mechanisms underlying invariant recognition of the arbitrary signs that compose quite distinct alphabets is a major step toward understanding reading. Particularly, a comparison of the process of letter recognition in Arabic versus Roman scripts may shed some light on whether the same dynamic processes underlie letter recognition in different alphabets (i.e., a hierarchical process in which higher-level processing becomes more and more abstract - as reflected in late components in the ERP waves) or whether some effects (e.g., the early visual effect found by Petit et al., 2006, in the Roman alphabet) are specific to the characteristics of a given alphabet. The combination of masked priming with ERPs in individuals who learned both the Arabic and Roman alphabet at an early age provides the means to capture potential early differential effects of visual similarity and later effects of abstract letter processing across alphabets, using a within-subject design.

Unlike the Petit et al. (2006) masked priming experiment in which the ERPs were measured in the absence of any task when the target letter appeared on the screen (except for occasional trials; $1 / 6$ of trials), we opted to employ a task (the masked priming same-different matching task) in which all trials required a response. This allows us to examine the relationships between the latency data and the ERP waves of early and late components. Furthermore, as indicated earlier, Petit et al. (2006) employed the 26 letters of the Roman alphabet. To examine the role of visual similarity, Petit et al. conducted a post hoc analysis of visual similarity in an early time window (180$220 \mathrm{msec}$ ). Here we opted for a direct manipulation of visual similarity. That is, we examined masked repetition priming for a subset of letters that were (1) visually similar/ dissimilar across case in the Roman alphabet (e.g., c-C vs. g-G) and (2) visually similar/dissimilar across letter position in the Arabic alphabet (e.g., ف ف vs. - - ع - ع). The general procedure of the experiment was parallel to that employed by Carreiras et al. (2012) and Kinoshita and Kaplan (2008). The main differences were that (i) ERPs were recorded, which allows us to separate the time course of visual and abstract letter representations, and (ii) the same participants were presented with Roman and Arabic letters-in different blocks.

Of specific interest in the present experiment are the early time windows in the ERP waves, in particular the N/ P150 component (see Petit et al., 2006). This component usually displays a posterior scalp distribution focused over right occipital scalp sites and seems to be sensitive to processing at the level of visual features. This can take the form of a bipolar effect across frontal and occipital electrodes. Whereas this component is more positive over occipital areas, it is more negative over frontal areas of the scalp (e.g., Chauncey, Holcomb, \& Grainger, 2008; Holcomb \& Grainger, 2006). We will also consider other components that occur slightly later in time, such as the N250 and the P3 components. The N250 component has been associated to the degree of prime-target orthographic and phonological overlap, thus suggesting that it is sensitive to processing sublexical representations (Carreiras, Duñabeitia, \& Molinaro, 2009; Grainger, Kiyonaga, \& Holcomb, 2006). The P3 component is usually present when the task requires a binary decision, and this component is normally associated with the response that participants have to execute (see Donchin \& Coles, 1988). Differences in the latency of the P3 have been found to vary as a function of the difficulty of stimulus evaluation (Kutas, McCarthy, \& Donchin, 1977). 


\section{METHODS}

\section{Participants}

Sixteen students (11 women) at Universidad Politécnica de Valencia and the Universitat de València took part in the experiment. Ages ranged between 20 and 30 years (mean $=23.5$ years). The participants were native speakers of Arabic who had attended primary and secondary school in their home countries. They reported having learnt the two alphabets (Arabic and Roman) very early in preschool/primary school. They also reported that, on a daily basis, they would read/write in both Modern Standard Arabic and Spanish (or languages which, like French/English, employ the Roman script). All of them had normal/corrected-to-normal vision and were righthanded, as assessed with an abbreviated version of the Edinburgh Handedness Inventory (Oldfield, 1971). The data of three of the participants were discarded because the raw EEG data were too noisy and presented too many artifacts to obtain stable data. Informed consent was obtained from all the participants. The ethical committee of the BCBL research center approved the study.

\section{Materials}

The experiment consisted of two blocks: one with Arabic letters and the other with Latin letters (i.e., Roman alphabet). In the Arabic block, we employed the same eight letters as Carreiras et al. (2012) and the same experimental design. Four of these letters were visually dissimilar when written in middle and isolated forms $(\Omega / \varepsilon$,

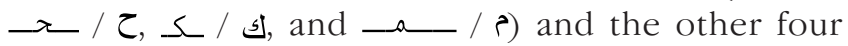
letters were visually similar when written in isolated and middle forms (bـ/ J). We created 32 probe-prime-target triplets in which the probe and the target were the same and 32 probeprime-target triplets in which the probe and the target were different. For "same" trials, we manipulated the prime-target relatedness (identity [e.g., Ccc], control [e.g., Cxc]) and the probe-target letter type (similar [e.g., Ccc or Cxc] dissimilar [e.g., Bbb or Bxb]); for "different" trials and to have a zero prime-target contingency (see Carreiras et al., 2012), we manipulated the probe-target relationship rather than the prime-target relationship. To avoid physical continuity, the prime was presented in Arial 12-point font, and the reference and the target were presented in Arial 22-point font. A list of examples for the different experimental conditions is displayed in Figure 1. To obtain stable data, we carried out the experimental block eight times (i.e., 512 trials overall; see also Carreiras et al., 2012, for a similar procedure).

In the Roman block, we also employed eight letters. Four letters were visually dissimilar when written in lowercase and uppercase (D/d, G/g, B/b, and R/r) and the other four letters were visually similar when written in lowercase and uppercase $(\mathrm{C} / \mathrm{c}, \mathrm{S} / \mathrm{s}, \mathrm{V} / \mathrm{s}$, and $\mathrm{X} / \mathrm{x})$-all these letters had been used by Kinoshita and Kaplan (2008). The creation of the lists was the same as in the Arabic block, except that the manipulation was case (lowercase vs. UPPERCASE) instead of letter position (middle vs. isolated). There were also 512 trials in this block. All participants received the two blocks: half of the participants received the Arabic block first, and the other half received the Roman block first.

\section{Procedure}

Participants were tested individually in a quiet room. Presentation of the stimuli and recording of behavioral/neurophysiological responses were controlled by Presentation software. On each trial, a reference letter was presented above a forward mask consisting of a hash mark (\#) for $1000 \mathrm{msec}$. Then the probe disappeared and the forward mask was replaced by a prime for $50 \mathrm{msec}$, and this was immediately replaced by the target stimulus. The target stimulus remained on the screen for 1000 msec or until the participant's response (see Figure 1 for a schematic representation of a given trial). The intertrial interval varied randomly between 500 and 1000 msec. After this interval, an asterisk was presented for 1000 msec to allow participants to blink. Participants were told that they would see two succeeding letters and that they were to press a button labeled "same" if they thought the two stimuli corresponded to the same letter and to press a button labeled "different" if they thought that the two stimuli were different letters. Participants were instructed to make this decision as fast and as accurately as possible. Participants reported not having seen any briefly presented stimuli (i.e., primes) when asked immediately after the experiment. Each participant received a different random order of stimuli. Each participant received a total of 20 practice trials before each experimental block.

\section{EEG Recording and Analyses}

Scalp voltages were collected using a BrainAmp recording system from $32 \mathrm{Ag} / \mathrm{AgCl}$ electrodes mounted in an elastic cap (ElectroCap International, Eaton, OH, 10-10 system). Figure 2 displays the schematic distribution of the recording sites. A left mastoid was used as on-line reference, and the signal was re-referenced off-line to the average of right and left mastoids before the statistical analyses. Eye movements and blinks were monitored with four further electrodes, thus providing bipolar recordings of the horizontal (Heog-, Heog+) and vertical (Veog-, Veog+) electrooculogram. Interelectrode impedances were kept below $5 \mathrm{~K} \Omega$. EEG was filtered with an analogue bandpass filter of $0.01-100 \mathrm{~Hz}$ and a digital 0.1 high-pass and $30 \mathrm{~Hz}$ low-pass filters were applied before the statistical analyses. The signals were sampled continuously throughout the experiment with a sampling rate of $500 \mathrm{~Hz}$.

As in prior research with the masked priming samedifferent matching task, the focus was exclusively on 


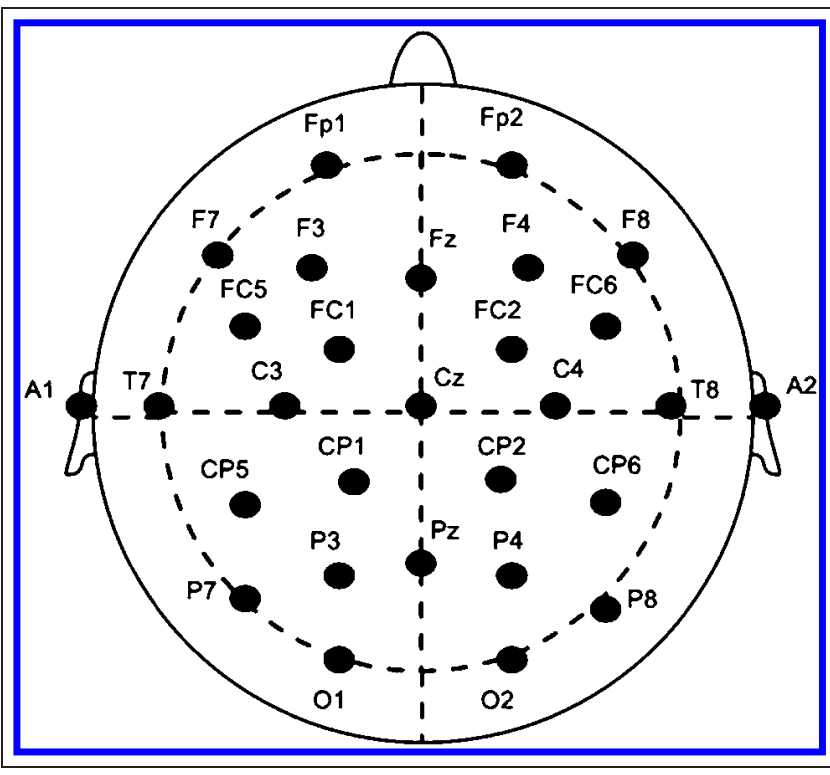

Figure 2. Electrode montage.

"same" responses. Bear in mind that, to keep a zero contingency scenario, "different" responses involved primes and targets that were unrelated (see Perea, Moret-Tatay, \& Carreiras, 2011, for discussion). When the probe and the prime were the same, in half of the trials the responses were "same" and in the other half they were "different." When the probe and the prime were different, in half of the trials the responses were "same" and in the other half they were "different." Only trials free of ocular artifacts (blinks and eye movements) and muscular artifacts were averaged and analyzed (more than 95\% of the trials). Epochs of the EEG up to $1000 \mathrm{msec}$ from the onset of the target letter were the primary data. The baseline correction was performed using the average EEG activity in the 100 msec preceding the onset of the target as a reference signal value. Separate ERPs were formed for each of the experimental conditions, each of the participants, and each of the electrode sites (see electrode numbers in Figure 2). We conducted an ANOVA considering the Electrode Factor (27), Prime-Target Relatedness (two levels: identity, control), Letter Type (two levels: similar, dissimilar), and Alphabet (two levels: Roman, Arabic). Neither the order of blocks nor the type of probe-target (lowercase-uppercase or uppercaselowercase in the Roman alphabet; medial-isolated or isolated-medial in the Arabic alphabet) had a significant effect on performance so that, for the sake of simplicity, they were not entered in the reported ANOVAs. Whenever an interaction with Electrode was found, ROIs were defined, which can reliably capture the distribution of effects. When appropriate, $p$ values are reported using the Greenhouse-Geisser (1959) correction. In accordance with our hypotheses, the statistical analyses focused on the main effects of Prime-Target Relatedness and their interactions with the other factors. An initial visual analy- sis was performed on the $0-1000$ msec time window, which was later divided into two windows (60-260 msec) and (280-500 msec). Peak amplitudes and peak latencies were obtained for different time windows.

\section{RESULTS}

\section{Behavioral Measures}

Incorrect responses and RTs that exceeded two standard deviations from the participant's mean were excluded from the latency analyses. The average RTs and percent errors are presented in Table 1. For "same" responses, ANOVAs based on the participant mean correct RTs and percentage of errors were conducted based on a 2 (PrimeTarget Relatedness: identity, control) $\times 2$ (Letter Type: similar, dissimilar) $\times$ Script (Roman vs. Arabic) .

The ANOVA on the RT data showed faster RTs for trials involving Roman letters than for trials involving Arabic letters, $F(1,12)=25.69, p<.001$, as well as faster RTs for trials involving "similar" letters than for the trials involving "dissimilar" letters, $F(1,12)=16.74, p<.001$. In addition, RTs were faster when the target letter was preceded by an identity letter prime than when preceded by a control letter prime, $F(1,12)=94.79, p<.001$. As expected, the repetition priming effect was quite large for both similar and dissimilar letters (48 vs. $37 \mathrm{msec}$, respectively, both $p s<.001)$ —note that the priming effect was $11 \mathrm{msec}$ greater for the similar letters than for the dissimilar letters (Similarity $\times$ Prime-Target Relatedness interaction, $F(1,12)=5.86, p=.032)$. Finally, the effect of Prime-Target Relatedness was not modulated by Script (two-way Relatedness $\times$ Script interaction: $F<1$; threeway Script $\times$ Relatedness $\times$ Similarity interaction: $F<1$ ).

The ANOVA on error data only revealed that participants committed more errors on control trials than on identity trials, $F(1,12)=10.26, p=.008$. None of the other effects/interactions were significant-note, however, that the relatedness effect was slightly greater (marginally significant) for the trials in the Roman script than for the trials in the Arabic script (two-way interaction: $F(1,12)=$ $3.88, p=.072$ ).

Table 1. Mean RTs and Percentage of Errors per Condition (SEs)

\begin{tabular}{lcccc}
\hline & \multicolumn{2}{c}{ Arabic Script } & \multicolumn{2}{c}{ Roman Script } \\
\hline Similar & & & & \\
Identity & $526(16.7)$ & $4.1(1.1)$ & $473(15.5)$ & $2.7(0.9)$ \\
Control & $573(13.5)$ & $6.2(1.3)$ & $521(13.0)$ & $5.8(1.7)$
\end{tabular}

\section{Dissimilar}

\begin{tabular}{lllll} 
Identity & $536(14.7)$ & $6.5(1.4)$ & $499(19.0)$ & $4.1(0.9)$ \\
Control & $570(14.4)$ & $7.2(1.6)$ & $538(15.2)$ & $7.0(1.1)$ \\
\hline
\end{tabular}




\section{Electrophysiological Measures}

For "same" responses, ERP grand averages in frontal and parietal electrodes time-locked to the onset of the target letters for the contrasts between the identity and control conditions for similar-dissimilar letters as well as the topographical maps of these effects are displayed in Figure 3. Visual inspection of Figure 3 reveals the presence of a consistent N/P150 component, with a frontal relatedness effect only when the prime is dissimilar to the target and a posterior effect when the prime is similar to the target. This effect is consistent across the two scripts.

In addition, an effect of prime-target relatedness appears at the P300 window (see Figure 4) with a centroparietal distribution for both similar and dissimilar letters and with a frontal distribution only for the dissimilar letters. Finally, there is a very salient difference in P300 latency depending on prime-target relatedness, regardless of visual similarity (see Figure 5). This faster latency for targets preceded by identity than for targets preceded by a control prime occurred both in Roman and Arabic scripts.

\section{Peak Amplitude}

N/P150. The polarity and distribution of the N/P150 depends on prime-target relatedness and on letter type. As shown in Figure 3, two ROIs were defined, including frontal (Fp1, Fp2, F7, F8, FC2, FC5, FC6) and posterior electrodes (CP5, P3, P4, P7, P8, O1). An ANOVA ROI (two levels: frontal and posterior) $\times$ Alphabet (two levels: Roman vs. Arabic) $\times$ Prime-Target Relatedness (two levels: identity and control) $\times$ Letter Type (two levels: similar vs. dissimilar) showed a Similarity $\times$ Relatedness interaction, $F(1,12)=5.48, p=.037$. To examine this interaction, we conducted ANOVAs separately for each condition of visual similarity. When probes and targets were visually similar, control prime-target pairs were

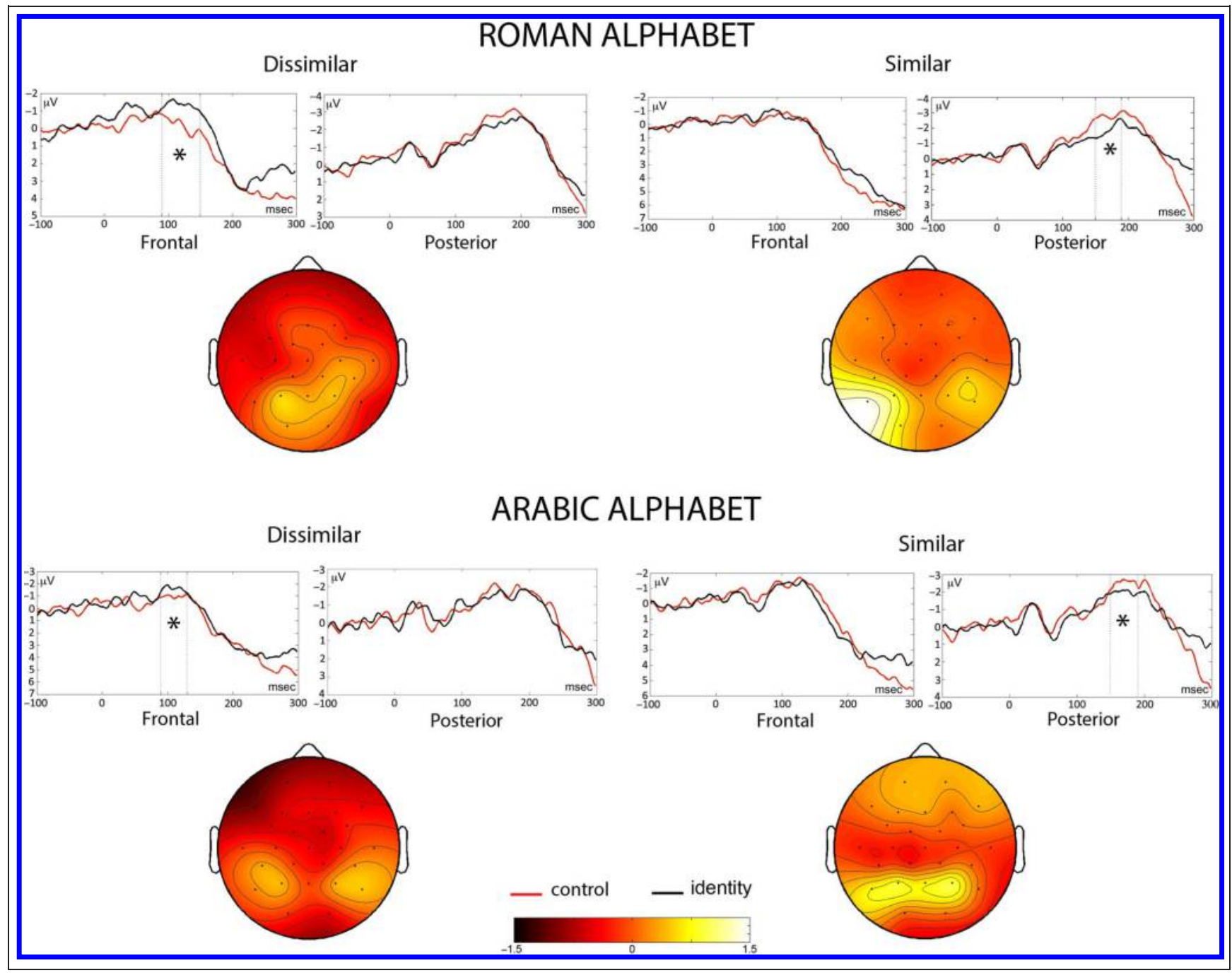

Figure 3. N1/P150 amplitude peak differences for the identity versus control contrast for similar and dissimilar letters in Arabic and Roman alphabets. Voltage maps represent differences in peak amplitudes, in $\mu \mathrm{V}$, and show different topographies depending on letter similarity. 
Figure 4. P3 amplitude peak differences for the identity versus control contrast. Peak amplitudes are computed at different latencies, and differences between identity and control conditions are plotted for each ROI. The three bars represent from left to right the three ROIs left/ central/right. Voltage maps represent differences in peak amplitudes (in $\mu \mathrm{V}$ ).

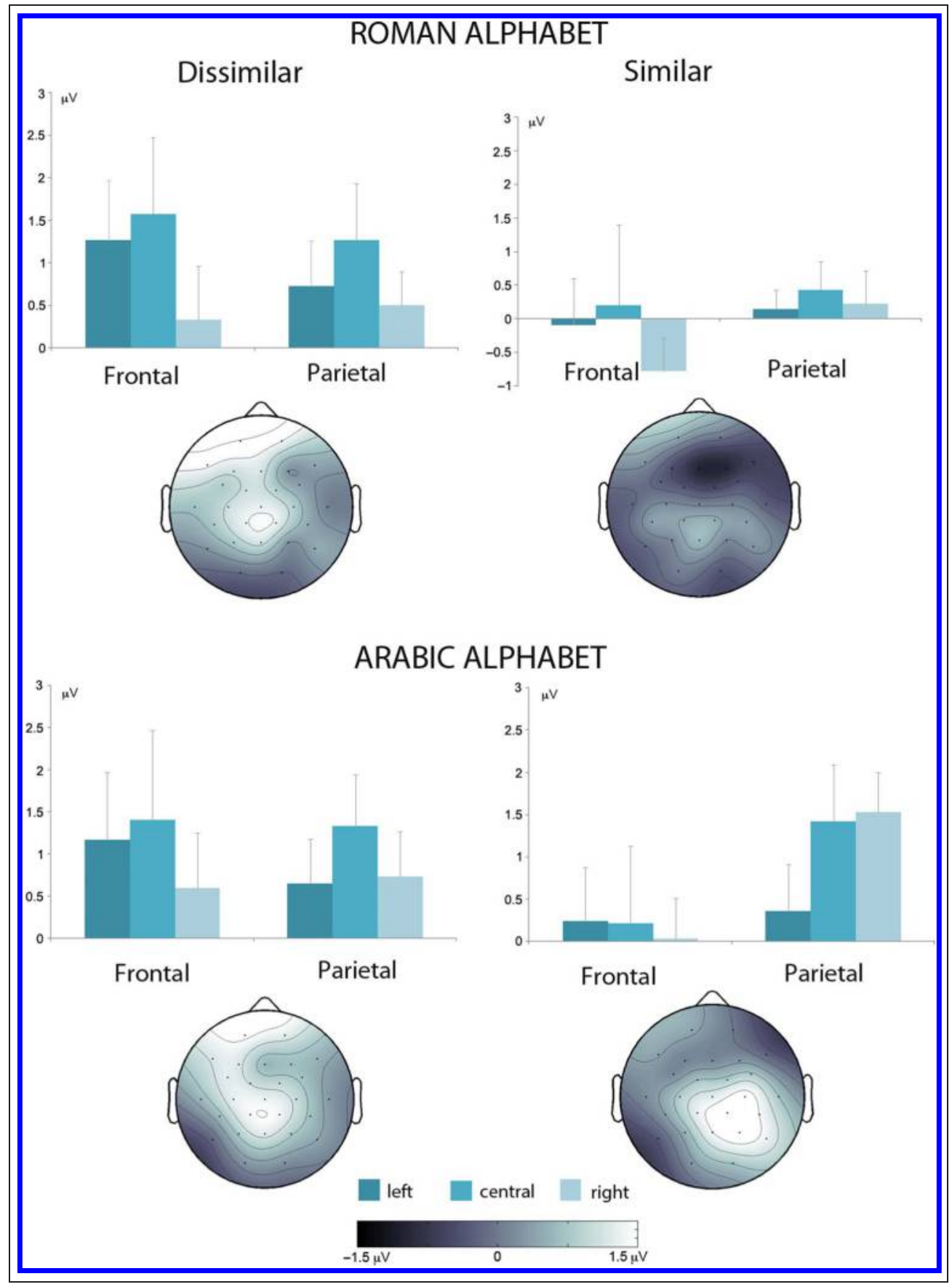

more negative than identity prime-target pairs in posterior electrodes, regardless of script (relatedness in frontal electrodes: $F<1$, relatedness in posterior electrodes: $F(1$, $12)=15.12, p=.002$; ROI $\times$ Relatedness: $F(1,12)=$ $3.75, p=.07)$. When probes and targets were visually dissimilar, control prime-target pairs were more positive than identity prime-target pairs in frontal electrodes, regardless of script (relatedness in frontal electrodes: $F(1$, $12)=7.87, p=.016$, relatedness in posterior electrodes: $F<1$; ROI $\times$ Relatedness: $F(1,12)=8.62, p=.012)$.

The N/P150 relatedness effect was measured by peak amplitude within an interval between 60 and $260 \mathrm{msec}$ (see Figure 3). Further analyses of consecutive time windows of 20 msec collapsing through the two alphabets showed that the relatedness effect started earlier for visually dissimilar than for visually similar letters (Time Window $\times$ Similarity $\times$ ROI $\times$ Relatedness: $F(5,60)=$ $6.06 ; p=.002$; see Table 2 ). The frontal effect for visually dissimilar letters appeared earlier (90-130 msec). In contrast, the parietal prime-target relatedness effect for visually similar letters appeared in the 150-190 msec time window after target onset.

P3 peak amplitude. Parietal classical distributed P3 effects and frontal P3 effects were analyzed separately. To better capture the effects in the frontal and parietal sites, 


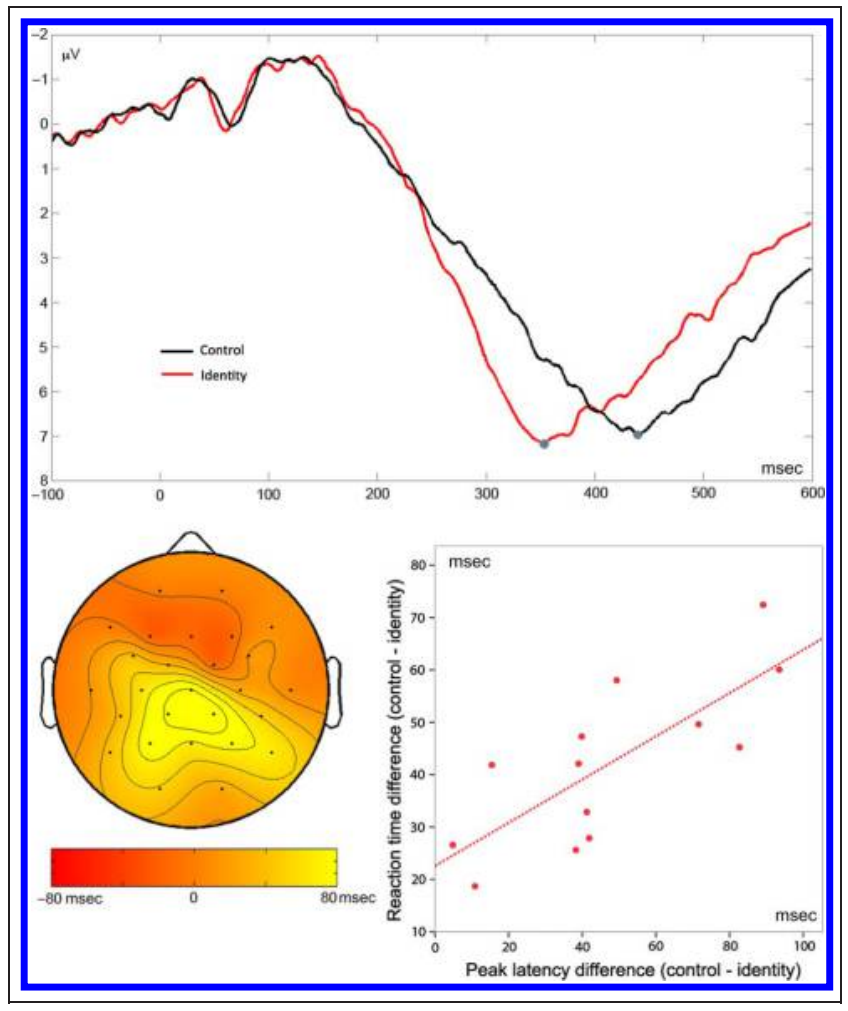

Figure 5. P3 peak latencies differences for identity versus control contrasts and regression between differences in RTs and differences in P3 latency. Voltage maps represent differences in peak latencies (in msec).

frontal sites were divided into three ROIs each (left-frontal: F7, F3; frontocentral: Fp1, Fp2; right-frontal: F8, F4). A similar ROIs division was applied for parietal sites (leftparietal: C3, CP5, P3; centroparietal electrodes: CP1, CP2, Pz; and right-parietal: C4, CP6, P4). The centroparietal peak amplitude showed a prime-target relatedness ef- fect, $F(1,12)=4.81, p=.049$, that did not depend on alphabet (Interaction Relatedness $\times$ Alphabet: $F(1,12)=$ $2.54, p=.1$ ) or on the visual similarity between probe and target (Interaction Letter Type $\times$ Relatedness: $F<1$ ).

Interestingly, the effects of relatedness on the P3 amplitude in frontal sites were modulated by similarity, appearing only for visually dissimilar probe-targets. For visually dissimilar probe-targets, an ROI $\times$ Relatedness interaction, $F(2,24)=6.1, p=.02$, showed that the relatedness effect was localized in fronto-central and left-frontal electrodes (central: $F(1,12)=5.18, p=.04$; left-frontal: 3.48, $p=.08$; collapsed: $F(1,12)=4.36, p=.05$; right-frontal: $F<1)$. No main effect of relatedness, $F(1,12)=0.003$, $p=.9$, or Relatedness $\times$ Alphabet interaction, $F(1,12)=$ $0.1, p=.6$, appeared for similar letters.

\section{Latencies}

N/P150 peak latencies. The peak latencies at this time window were not modulated by any of the experimental factors.

P3 latency. The P3 latency was modulated by relatedness, but not by probe-target visual similarity. All sites with the exception of most frontal electrodes (Fp1, Fp2, F4, F7, F8) showed earlier peak latencies for identity primetarget, $F(1,12)=27.98, p<.001$. This effect was independent of visual similarity between prime and target.

P300 prediction of RTs. A final analysis revealed that the P3 latency correlated strongly with the priming effects in the latency data $(r=.79, p=.001)$ : The greater the difference between the control and identity conditions in the P300 peak latency, the greater the priming effect in the latency data.

Table 2. Amplitude Differences between the Repetition Priming and Unrelated Condition (Relatedness Effect) for Similar and Dissimilar Letters in the Two Alphabets Together in the Two ROIs (Frontal and Parietal) for Windows of 20 msec in the P/N150

\begin{tabular}{|c|c|c|c|c|c|c|}
\hline & $90-110 \mathrm{msec}$ & $110-130 \mathrm{msec}$ & $130-150 \mathrm{msec}$ & $150-170 \mathrm{msec}$ & $170-190 \mathrm{msec}$ & $190-210 \mathrm{msec}$ \\
\hline \multicolumn{7}{|c|}{ Dissimilar } \\
\hline \multirow[t]{2}{*}{ Frontal } & $F(1,12)=5.8$ & $F(1,12)=5.81$ & $F(1,12)=2.09$ & $F(1,12)=3.7$ & $F(1,12)=1.8$ & $F(1,12)=0.06$ \\
\hline & $p=.03$ & $p=.03$ & $p=.05$ Roman & $p=.07$ & $p=.2$ & $p=.8$ \\
\hline \multirow[t]{2}{*}{ Parietal } & $F(1,12)=0.68$ & $F(1,12)=1.15$ & $F(1,12)=4.45$ & $F(1,12)=1.29$ & $F(1,12)=1.8$ & $F(1,12)=0.04$ \\
\hline & $p=.4$ & $p=.3$ & $p=.06$ & $p=.27$ & $p=.19$ & $p=.8$ \\
\hline \multicolumn{7}{|l|}{ Similar } \\
\hline \multirow[t]{2}{*}{ Frontal } & $F(1,12)=0.08$ & $F(1,12)=0.76$ & $F(1,12)=0.27$ & $F(1,12)=1.45$ & $F(1,12)=0.2$ & $F(1,12)=0.02$ \\
\hline & $p=.7$ & $p=.4$ & $p=.61$ & $p=.25$ & $p=.6$ & $p=.8$ \\
\hline \multirow[t]{2}{*}{ Parietal } & $F(1,12)=0.4$ & $F(1,12)=0.1$ & $F(1,12)=0.54$ & $F(1,12)=6.98$ & $F(1,12)=5.5$ & $F(1,12)=3.01$ \\
\hline & $p=.5$ & $p=.7$ & $p=.47$ & $p=.02$ & $p=.03$ & $p=.1$ \\
\hline
\end{tabular}

The shaded areas mark the effects that were significant in the different time windows. 


\section{DISCUSSION}

The present experiment has revealed a number of relevant findings for neural models of letter and word recognition. First, as in previous behavioral experiments with the Roman and Arabic alphabets, RTs were faster when the target letter was preceded by an identity letter prime than when preceded by an control letter prime-note that the priming effect was slightly larger for visually similar than for visually dissimilar pairs. Second, we found a consistent N/P150 component. This component revealed a frontal relatedness effect when primes and targets were visually dissimilar and a posterior effect when they were visually similar. In addition, the effect for visually dissimilar targets occurred in an earlier time window than that for visually similar targets. Again, this effect was equivalent in the Roman and Arabic alphabets. Third, we obtained a main effect of prime-target relatedness in the P300 time window with a centro-parietal distribution for both visually similar and dissimilar prime-target pairs and with a frontal distribution only for the visually dissimilar letters-again this effect was similar in the two scripts. Fourth, there was a very salient difference of prime-target relatedness in P3 latency that was not modulated by letter similarity-this effect also occurred similarly in the Roman and Arabic scripts. And fifth, the latency of the P3 component strongly predicted RTs.

Thus, the present data have revealed that processing of letters in the Roman and Arabic scripts follows approximately the same path. The similarities between the processing of letters in the two alphabets is particularly striking if we consider that in the Roman alphabet we manipulated case (uppercase vs. lowercase) whereas in the Arabic alphabet we used primes and targets that exhibit different forms depending on their position in a word (middle vs. isolated). This strongly suggests that the fast conversion from visual form into visual formindependent abstract letter representations is a universal phenomenon, that is, it occurs in different alphabets. Therefore, the present data are consistent with neural accounts that propose that the time course of letter processing involves sensitivity to physical similarity in the earlier stages of processing, followed by an abstract identification of letters in later stages of processing (e.g., Grainger et al., 2008; Dehaene et al., 2005). This study has established that these accounts, proposed initially for the Roman script, can be readily generalized to other alphabets (Arabic).

Similar to previous behavioral studies with the masked priming same-different matching task, we found a masked repetition priming effect in Roman and Arabic for letter pairs with similar visual features and for letter pairs with dissimilar visual features (Carreiras et al., 2012; Kinoshita \& Kaplan, 2008). Although the effect was robust for both visually similar and visually dissimilar letter pairs, it was slightly larger for the visually similar pairs (interaction: $p=.03)$. This may have been because of the fact that the statistical power was larger than in previous experiments (i.e., number of trials was larger), and it may represent a residual effect of visual similarity in the RTs. This may be analogous to the residual effect of mirror invariance for reversible letters that occurs in the early stages of processing (Duñabeitia, Molinaro, \& Carreiras, 2011). Despite the fact that we develop the capacity of inhibiting mirror invariance for reading and achieve an abstract representation of letters (e.g., Pegado, Nakamura, Cohen, \& Dehaene, 2011; Perea, Moret-Tatay, \& Panadero, 2011; Dehaene et al., 2005, 2010; see Kolinsky et al., 2011, for a review), the cognitive system may still retain some sensitivity to the effects of visual form.

The analyses of the ERP signal revealed a relatedness effect for the visually dissimilar targets in the 90-130 msec time window in frontal electrodes, whereas for the visually similar targets the effect occurred in the 150-190 msec time window in parietal electrodes - note that the polarity of the two effects was the opposite. Interestingly, equivalent effects were observed in the two alphabets. This pattern of data suggests an early contribution of letter form sensitivity (i.e., the effects for visually dissimilar letters are slightly earlier than for visually similar letters) followed by an abstract letter identification processes. There is an effect of repetition priming for both similar and dissimilar letters that occur within a 200-msec window that is consistent with previous masked priming experiments (see Petit et al., 2006), and this also suggests that the P/N150 component, regardless of its precise topography and polarity, is sensitive to letter identification processes (see Wong et al., 2005; Tarkiainen, Cornelissen, \& Salmelin, 2002; Tarkiainen, Helenius, Hansen, Cornelissen, \& Salmelin, 1999; Schendan, Ganis, \& Kutas, 1998; Bentin et al., 1996).

In a later window (280-500 msec) signaling the P3 component, we found a masked repetition priming effect of equivalent magnitude for visually similar and dissimilar letters with a centro-parietal distribution that also occurred for dissimilar letters with a frontal distribution. This reveals the existence of an abstract processing of the letters, but it also reveals that (to some degree) this process is modulated by visual similarity even at later processing stages. It is important to note here that the relatively late ERP activity related to letter processing has also been reported in previous experiments (Madec et al., 2012; see Petit et al., 2006). Madec et al. interpreted this late activity as feedback processing to amplify and stabilize the activity in the primary areas to help letter recognition (see also Twomey, Kawabata, Price, \& Devlin, 2011). Another possibility is that the P300 (P3) component that has been elicited with a frontal distribution by the dissimilar letters resembles the $\mathrm{P} 3 \mathrm{a}$ component. The $\mathrm{P} 3 \mathrm{a}$ component has been associated with brain activity related to the engagement of attention and the processing of novelty (see Polich, 2003). Thus, control primes may attract more attention/be more novel in the case of dissimilar letters when comparing probes and targets and thus still show sensitivity to the 
visual appearance of the primes. In contrast, it could be argued that the P3 component with the centro-parietal distribution for visually dissimilar and similar letters resembles the so-called P3b component, which is thought to reflect processes involved in stimulus evaluation, categorization, decision-making, and/or updating (Donchin \& Coles, 1988; Donchin, 1981), or an index of the match or mismatch of the target as compared with a maintained working memory trace (Näätänen, 1990) that in the present experiment would be the one generated by the probe. Specifically, according to Näätänen's view, the prime in the identity priming condition would match the memory trace established between the probe and the target, whereas the unrelated prime would deviate from them, thus leading to the observed P3 effect. Importantly, this finding (which occurs both in the Roman and Arabic alphabets) also supports fast conversion to an abstract processing of the letters.

A final finding that deserves consideration is that the relatedness effects obtained in the $\mathrm{P} 3$ component were closely related to the relatedness effects obtained in the RTs (see McCarthy \& Donchin, 1981). As indicated earlier, statistical analyses of the peak P3 latencies revealed a repetition priming effect for visually similar and dissimilar letters in the two alphabets. The latency of the P3 component strongly predicted RTs. Given that differences in latency of the P3 have been found to vary as a function of the difficulty of stimulus evaluation, they may reflect the time it takes to evaluate/categorize events (e.g., McCarthy \& Donchin, 1981; Kutas et al., 1977). Therefore, the longer latency of the P300 elicited by the unrelated control condition relative to the identity condition may well be the result of a longer processing time needed to categorize whether the probe and target are the same when the prime is an unrelated control item.

In summary, the present ERP data, obtained in two very distinct alphabets (Roman and Arabic) are consistent with a universal model of letter recognition that includes the activation of abstract representations during the late processes corresponding to letter identification but that retains some form of sensitivity during the entire flow of information processing.

\section{Acknowledgments}

The research reported in this article has been partially supported by grants CONSOLIDER-INGENIO2010, CSD2008-00048, and PSI2011-26924 from the Spanish Ministry of Economy and Competitiveness and grant ERC-2011-ADG-295362 from the European Research Council.

Reprint requests should be sent to Manuel Carreiras, Basque Center on Cognition, Brain, and Language, Paseo Mikeletegi, 69, 20009, Donostia-San Sebastián, Spain, or via e-mail: m.carreiras@bcbl.eu.

\section{REFERENCES}

Bentin, S., Allison, T., Puce, A., Perez, A., \& McCarthy, G. (1996). Electrophysiological studies of face perception in humans. Journal of Cognitive Neuroscience, 8, 551-565.
Bowers, J. S., Vigliocco, G., \& Haan, R. (1998). Orthographic, phonological, and articulatory contributions to masked letter and word priming. Journal of Experimental Psvchologv: Human Perception \& Performance, 24, 1705-1719.

Carreiras, M., Duñabeitia, J. A., \& Molinaro, N. (2009). Consonants and vowels contribute differently to visual word recognition: ERPs of relative position priming. Cerebral Cortex, 19, 2659-2670.

Carreiras, M., Perea, M., \& Abu Mallouh, R. (2012). Priming of abstract letter representations may be universal: The case of Arabic. Psychonomic Bulletin and Review, 19, 685-690.

Chauncey, K., Holcomb, P. J., \& Grainger, J. (2008). Effects of stimulus font and size on masked repetition priming: An ERP investigation. Language and Cognitive Processes. 23, 183-200.

Dehaene, S., Cohen, L., Sigman, M., \& Vinckier, F. (2005). The neural code for written words: A proposal. Trends in Cognitive Sciences, 9, 335-341.

Dehaene, S., Nakamura, K., Jobert, A., Kuroki, C., Ogawa, S., \& Cohen, L. (2010). Why do children make mirror errors in reading? Neural correlates of mirror invariance in the visual word form area. Neuroimage, 49, 1837-1848.

Donchin, E. (1981). Surprise! ... Surprise? Psychophvsiology, 18, 493-513.

Donchin, E., \& Coles, M. G. H. (1988). Is the P300 component a manifestation of context updating? Behavioral and Brain Sciences, 11, 355-372.

Duñabeitia, J. A., Molinaro, N., \& Carreiras, M. (2011). Through the looking-glass: Mirror reading. Neuroimage, 54 , 3004-3009.

Forster, K. I., \& Davis, C. (1984). Repetition priming and frequency attenuation in lexical access. Journal of Experimental Psychology: Learning. Memorv. and Cognition, 10, 680-698.

Grainger, J., Kiyonaga, K., \& Holcomb, P. J. (2006). The time course of orthographic and phonological code activation. Psychological Science, 17, 1021-1026.

Grainger, J., Rey, A., \& Dufau, S. (2008). Letter perception: From pixels to pandemonium. Trends in Cognitive Sciences, 12, 381-387.

Greenhouse, S., \& Geisser, S. (1959). On methods in the analysis of profile data. Psvchometrika, 24, 95-112.

Holcomb, P. J., \& Grainger, J. (2006). On the time-course of visual word recognition: En ERP investigation using masked repetition priming. Lournal of Cognitive Neuroscience, 18, $1631-1643$

Kinoshita, S., \& Kaplan, L. (2008). Priming of abstract letter identities in the letter match task. Ouarterly Iournal of Experimental Psvchologv, 61, 1873-1885.

Kolinsky, R., Verhaeghe, A., Fernandes, T., Mengarda, E. J., Grimm-Cabral, L., \& Morais, J. (2011). Enantiomorphy through the looking glass: Literacy effects on mirror-image discrimination. Journal of Experimental Psychology: General, 140, 210-238.

Kutas, M., McCarthy, G., \& Donchin, E. (1977). Augmenting mental chronometry: The P300 as a measure of stimulus evaluation time. Science, 197, 792-795.

Madec, S., Rey, A., Dufau, S., Klein, M., \& Grainger, J. (2012). The time course of visual letter perception. Iournal of Cognitive Neuroscience, 24, 1645-1655.

McCarthy, G., \& Donchin, E. (1981). A metric for thought: A comparison of P300 latency and response time. Science, 211, 77-80.

Näätänen, R. (1990). The role of attention in auditory information processing as revealed by event-related potentials and other brain measures of cognitive function. Behavioral and Brain Sciences, 13, 201-288. 
Oldfield, R. C. (1971). The assessment and analysis of handedness: The Edinburgh inventory. Neuropsvchologia. 9, 97-113.

Pegado, F., Nakamura, K., Cohen, L., \& Dehaene, S. (2011). Breaking the symmetry: Mirror discrimination for single letters but not for pictures in the visual word form area. Neuroimage, 55, 742-749.

Perea, M., Abu Mallouh, R., \& Carreiras, M. (2013). Early access to abstract representations in developing readers: Evidence from masked priming. Developmental Science, 16, 564-573

Perea, M., Moret-Tatay, C., \& Carreiras, M. (2011). Facilitation vs. inhibition in the masked priming same-different matching task. Ouarterlv Iournal of Experimental Psvchology, 64, 2065-2079.

Perea, M., Moret-Tatay, C., \& Panadero, V. (2011). Suppression of mirror generalization for reversible letters: Evidence from masked priming. Iournal of Memory and Language. 65, 237-246.

Petit, J. P., Midgley, K. J., Holcomb, P. J., \& Grainger, J. (2006). On the time-course of letter perception: A masked priming ERP investigation. Psvchonomic Bulletin and Review, 13, 674-681.

Polich, J. (2003). Overview of P3a and P3b. In J. Polich (Ed.), Detection of change: Event-related potential and fMRI findings (pp. 83-98). Boston: Kluwer Academic Press.
Rey, A., Dufau, S., Massol, S., \& Grainger, J. (2009). Testing computational models of letter perception with item-level ERPs. Cognitive Neuropsvchology, 26, 7-22.

Schendan, H. E., Ganis, G., \& Kutas, M. (1998). Neurophysiological evidence for visual perceptual categorization of words and faces within $150 \mathrm{~ms}$. Psychophysiology, 35, 240-251.

Tarkiainen, A., Cornelissen, P. L., \& Salmelin, R. (2002). Dynamics of visual feature analysis and object-level processing in face versus letter-string perception. Brain, 125, 1125-1136.

Tarkiainen, A., Helenius, P., Hansen, P. C., Cornelissen, P. L., \& Salmelin, R. (1999). Dynamics of letter string perception in the human occipitotemporal cortex. Brain, 122, 2119-2131.

Twomey, T., Kawabata, D. K. J., Price, C. J., \& Devlin, J. T. (2011). Top-down modulation of ventral occipito-temporal responses during visual word recognition. Neuroimage, $55,1242-1251$

Whitney, C. (2001). How the brain encodes the order of letters in a printed word: The SERIOL model and selective literature review. Psvchonomic Bulletin and Review, 8, 221-243.

Wong, A. C., Gauthier, I., Woroch, B., DeBuse, C., \& Curran, T. (2005). An early electrophysiological response associated with expertise in letter perception. Cognitive. Affective $\mathcal{E}$ Behavioral Neuroscience, 5, 306-318. 